\title{
Aknemittel bremst MS nach erstem Schub
}

\section{Das Antibiotikum Minocyclin verzögert bei Patienten mit einem ersten Krankheitsschub den Übergang in eine klinisch gesicherte MS. In einer Phase- III-Studie wurde die Konversionsrate im Vergleich zu Placebo fast halbiert.}

$\mathrm{O}$ ffenbar lassen sich mit dem günstigen und seit langem generisch erhältlichen Antibiotikum Minocyclin bei Patienten nach einem ersten demyelinisierenden Ereignis (klinisch isoliertes Syndrom, CIS) erneute Schübe verhindern. Dies wird aus Daten einer PhaseIII-Studie deutlich, die Dr. Luanne Metz, von der Universität in Calgary/Kanada, vorstellte. Die Resultate könnten für Länder bedeutsam sein, in denen Patienten nach wie vor erst dann eine immunmodulierende Therapie erhalten, wenn sie einen zweiten Schub entwickeln. Nach ersten Daten sei das Antibiotikum auch in Kombination mit oralen Immunmodulatoren wirksam und ließe sich künftig vielleicht als Zusatztherapie einsetzen, so Metz.

In der Studie waren 143 Patienten entweder mit Minocyclin oder Placebo behandelt worden. Alle Patienten hatten in den sechs Monaten vor Studienbeginn ein erstes demyelinisierendes Ereignis. Die Teilnehmer waren im Schnitt 36 Jahre alt, knapp $70 \%$ hatten zu Beginn bereits mehr als acht T2-Läsionen. Behandelt wurde entweder so lange, bis nach den alten McDonald-Kriterien von 2005 eine MS diagnostiziert werden konnte, oder 24 Monate, wenn bis dahin keine weiteren Schübe auftraten. Primärer Endpunkt war die MS-Inzidenz nach sechs Monaten. In der MinocyclinGruppe bekamen die Patienten - wie auch in der Aknetherapie üblich - zweimal täglich 100 mg der Substanz.

\section{MS-Inzidenz um $45 \%$ geringer}

Das überraschende Ergebnis: In den ersten sechs Monaten nach Studienbeginn konvertierten $61 \%$ unter Placebo, aber nur $34 \%$ unter Minocyclin zu einer MS. Das Erkrankungsrisiko wurde damit knapp um die Hälfte (45\%) reduziert; die absolute Risikoreduktion lag bei $27 \%$. Nach einem Jahr war das Ergebnis allerdings nicht mehr ganz so gut, die relative Risikoreduktion im Vergleich $\mathrm{zu}$ Placebo lag noch bei $38 \%$, die absolute bei $25 \%$, der Unterschied blieb aber signifikant $(\mathrm{p}=0,002)$.

Aufgrund der geringen Kosten und des günstigen Nebenwirkungsprofils sei Minocyclin für die Erstbehandlung bei CIS durchaus interessant, so Metz. Auch sollten künftige Studien den Nutzen zur Add-on-Behandlung in Kombination mit Immunmodulatoren überprüfen.

Für Minocyclin konnten entzündungshemmende Effekte nachgewiesen werden. So scheint der Wirkstoff die Durchlässigkeit der Blut-Hirn-Schranke für autoaggressive T-Lymphozyten zu reduzieren, tierexperimentelle Studien deuten zudem auf neuroprotektive Eigenschaften. Das Antibiotikum wird derzeit auch bei einigen neurodegenerativen Erkrankungen geprüft.

Thomas Müller

Parallel Session 13: Late breaking news,

31. ECTRIMS-Kongress, Barcelona, 7. - 10.10.2015

\section{Stammzelltherapie hält MS meist nur fünf Jahre in Schach}

Wenn sonst nichts mehr hilft, dann oft noch die Stammzelltherapie. Jedoch kehrt die MS bei der Hälfte der Behandelten innerhalb von fünf Jahren wieder zurück. Am ehesten profitieren junge Patienten mit wenigen Behinderungen.

$\mathrm{D}$ ie Myeloablation mit anschließender Transplantation autologer Stammzellen wird als Ultima Ratio bei Patienten mit sehr schwer verlaufender MS angeboten - also solchen, die trotz MS-Medikation eine hohe Schubfrequenz und Läsionslast oder eine rasche Krankheitsprogression aufweisen. Die Methode kann zwar die Krankheitsaktivität bei den meisten Patienten über Jahre hinweg zum Stillstand bringen, ist aber mit erheblichen Nebenwirkungen verbunden, meist sind diese hämatopoetischer oder gastrointestinaler Natur, zudem wurden Embolien und Todesfälle beschrieben. In früheren Untersuchungen lag die
Sterberate bei $5-6 \%$, mit neueren Chemotherapeutika sind es deutlich weniger.

Aufgrund der Risiken möchten viele Patienten wissen, wie lange ein solcher radikaler Reset des Immunsystems anhält. Langzeitdaten gibt es jedoch kaum. Nach den Ergebnissen der sehr kleinen Phase-II-Studie HALT-MS $(\mathrm{n}=24)$ scheint nach fünf Jahren wieder die Hälfte eine gewisse MS-Aktivität zu entwickeln. Dies wird nun durch eine größere Registeranalyse bestätigt. Für die Analyse hat eine Team um Dr. Paolo Muraro vom Imperial College in London weltweit sämtliche größere Zentren angeschrieben, die MS-Patienten mit autolo- gen Stammzellen behandeln. Auf diese Weise erhielten sie Daten zu 281 Patienten aus 25 Zentren - das sind $57 \%$ aller registrierten MS-Patienten mit Stammzelltransplantation. Die Patienten waren zum Zeitpunkt der Behandlung im Schnitt 37 Jahre alt, seit rund sieben Jahren erkrankt, hatten einen EDSS-Wert von 6,5 Punkten und litten zum größten Teil an einer chronisch-progredienten Form der Erkrankung (78\%).

\section{Nach zehn Jahren nur $30 \%$ progressionsfrei}

Für 112 Patienten liegen bereits 5-JahresDaten vor. Von diesen zeigten $49 \%$ nach fünf Jahren noch keine klinische Krankheitsprogression (keine Schübe, keine Behinderungsprogression). 31 Patienten konnten über mindestens zehn Jahre nachbeobachtet werden. Von diesen waren nur noch rund $30 \%$ progressionsfrei. Insgesamt scheinen jüngere Patienten von der Therapie deutlich stärker zu profitieren - von den 18- bis 30-Jährigen 